\title{
Lumen
}

Selected Proceedings from the Canadian Society for Eighteenth-Century Studies

\section{Le secret exposé selon Arlequin poli par l'amour (1720)}

\section{Servanne Woodward}

Volume 37, 2018

URI : https://id.erudit.org/iderudit/1042220ar

DOI : https://doi.org/10.7202/1042220ar

Aller au sommaire du numéro

Éditeur(s)

Canadian Society for Eighteenth-Century Studies / Société canadienne d'étude du dix-huitième siècle

ISSN

1209-3696 (imprimé)

1927-8284 (numérique)

Découvrir la revue

Citer cet article

Woodward, S. (2018). Le secret exposé selon Arlequin poli par l'amour (1720).

Lumen, 37, 1-16. https://doi.org/10.7202/1042220ar d'utilisation que vous pouvez consulter en ligne.

https://apropos.erudit.org/fr/usagers/politique-dutilisation/ 


\title{
Le secret exposé selon Arlequin poli parl'amour (1720)
}

\author{
SERVAnne WoOdWARd \\ University of Western Ontario
}

Une fée qui est à la veille d'épouser Merlin l'enchanteur enlève un «beau brun ${ }^{1}$ » et l'ayant enfermé dans son château, elle cherche à le séduire. Cette opération reste sans succès. Elle se rend compte que du côté d'Arlequin, il existe un obstacle. Pour s'en saisir elle utilise son anneau qui la rend invisible, ce qui lui permet de découvrir l'existence de Silvia, la bergère qui monopolise son affection. Sous menace expresse de la fée de tuer Arlequin si Silvia ne le chasse, la bergère prend rendez-vous pour persuader le jeune homme de la quitter. La question est de savoir si la fée est présente à cette rencontre sous le sceau de l'invisibilité, comme elle l'a promis à Silvia, ou absente, comme elle l'a promis à Arlequin. Sans aller jusqu'au panoptique de Foucault, la possibilité permanente de cette surveillance, combinée à l'amour qu'Arlequin ressent pour la première fois, lui a donné de l'esprit, donc des capacités de dissimulation de son cru.

Le conte de fée «Le parfait amour » (1698) de Mme de Murat peut avoir inspiré cette pièce ${ }^{2}$. Après avoir tué son frère, la fée Danamo a

1. L'expression est de Trivelin, sc. 1, dans Pierre Carlet de Chamblain de Marivaux, Théâtre complet, éd. de Frédéric Deloffre et Françoise Rubellin, Paris, Garnier frères, 1981, vol. I, p. 87 .

2. Henriette-Juliette de Castelnau, Mme de Murat, «Le parfait amour», dans Nouveau cabinet des fées. Les nouveaux contes de fées par madame la Comtesse de Murat, Genève, Slatkine Reprint, 1978 [réimpression de l'édition de Paris, 1765-1786,], t. 1, p. 202-248. Dans leur notice, Deloffre et Rubellin mentionnent aussi le conte de fées de Mme Durand, «Le prodige d'amour », qui a été identifié par Shirley E. Jones, et où Brutalis «marchait toujours pesamment, sa tête était penchée, et ses regards toujours fixés à terre », un bel homme qui résiste vaillamment à toutes les entreprises 
pris possession de son très jeune neveu, Parcin Parcinet, qu'elle élève avec le projet de lui faire épouser sa fille (laide et méchante, comme sa mère). Quand vient le temps du mariage, le jeune homme a un mouvement de déplaisir qu'il apprend immédiatement à dissimuler: «[il] compri[t] l'imprudence de ce qu'il venoit de faire, appela son esprit au secours de son cœur ${ }^{3} » ;$ «Parcin Parsinet [...] avoit enfin pris la résolution de se contraindre pour ne pas rendre inutile ses desseins $[\ldots]^{4} »$. Il est amoureux d'Irolite, une autre de ses cousines, qui vit emprisonnée et isolée au bout du jardin, derrière un lac, et à laquelle il rend visite à l'insu de Danamo. La surveillance permanente et imperceptible est un thème de ce conte: «Allez prince, lui dit-elle; que savons-nous si la fée n'a point ici quelqu'un prêt à lui rendre compte de ce qui s'y passe ${ }^{5}$ ?». Il existe un anneau magique qui fait disparaître son possesseur lorsqu'il le souhaite, mais c'est Parcin qui l'a reçu d'une fée qui lui est favorable, et son effet est limité à quatre souhaits. Il s'avère inutile à l'élargissement des deux prisonniers dont la fuite échoue. Parcinet et Irodite sont libérés et s'épousent par un enchaînement de circonstances improbables impliquant le sentiment d'amour.

Létude qui suit tend moins à chercher si Marivaux aurait puisé dans la vogue des contes de fée du siècle précédent, et donc à faire une étude comparative de Murat et Marivaux, que d'éclairer quelques aspects du secret comme archétype, ce dernier ayant pour contrepartie la surveillance, et donc sans doute un ethos du comportement de l'être en ce qu'il règle la liberté intime par rapport au contrat social «natu-

de séduction de la fée Coquette jusqu'à ce qu'il rencontre sa «bergère » et apprenne les bonnes manières et l'usage de la parole, ce qui lui vaut le sobriquet de «Polidamour » ("Notice», dans Marivaux, Théâtre complet, op. cit., p. 74-75). Ils se réfèrent à l'article de Jones, "A Probable Source of Marivaux's Arlequin poli par l'amour», French Studies: A Quarterly Review, 19, 1965, p. 385-391, et au conte de Mme Catherine Bédacier Durand, «Le prodige d'amour », accessible sur le site de Christine Rousseau à <http://www.lescontesdefees.fr/contes-et-auteurs/mme-durand/le-prodige-damour/>. Deloffre et Rubellin signalent également que Chimon, de la première des cinquièmes journées du Décaméron de Boccace, est le prototype d’Arlequin («Notice», dans Marivaux, Théâtre complet, op. cit., p. 78). Lucette Desvignes signale aussi une fable de Dryden, Cymon et Iphigénie, dans « Une source nouvelle d'Arlequin poli par l'amour", Revue des sciences humaines, 105, 1962, p. 31-38.

3. Mme de Murat, «Le parfait amour », op. cit., p. 206.

4. Ibid., p. 221.

5. Ibid., p. 218. 
rel », c'est-à-dire «universellement» reconnu, quel que soit le régime particulier considéré. Le merveilleux serait donc ici un lieu plus ou moins abstrait, parallèle au monde véritable, et permettant sinon une fiction des origines, la possibilité de cerner la question du secret et de ses développements connexes. Arlequin serait un ingénu du secret, nous en livrant les rouages à découvert $t^{6}$. Comme Silvia cherche à quoi reconnaître l'amour, nous sommes à même de reconnaître ce qui éveille la surveillance toujours plus ou moins clandestine. Avec cette pièce, nous en sommes presque à identifier les droits inaliénables des sujets qui ne peuvent être emprisonnés sans raison justifiable - une des compréhensions de l'Habeas corpus ${ }^{7}$.

Dans les deux cas, le pouvoir de la mère-fée, qui se substitue aux parents du jeune homme enlevé, relève de l'effraction. Danamo a tué son frère et sa sœur pour s'approprier leurs terres et leurs enfants. La fée de Marivaux satisfait un désir illégitime et inavouable. Sans doute la mère a-t-elle tout pouvoir sur le corps de l'enfant puisque dans la pièce de Marivaux, elle nourrit, distrait, loge un personnage qui ne s'exprime que par des cris ou des mots indicatifs de besoin. D’une certaine manière, Marivaux s'accorde avec Rousseau qu'à l'origine des langues est bien la nécessité d'exprimer «aidez-moi ». «Aimez-moi » vient cependant rapidement en second plan, lors de l'appel d'Arlequin à Silvia, quand il demande à la revoir. La parole articulée et discursive naît de la passion, mais une passion rationnelle et relationnelle. Sa passion est donc mesurée par la réponse de Silvia, et de surcroît, elle est contrôlée, menteuse s'il le faut, à cause de l'interférence qu'il détecte en la fée. Car le langage qu'il vient d'acquérir le portant dans la relation à autrui, l'expose aussi à l'épreuve des oreilles et des yeux des autres, projetant Arlequin dans la prise de conscience qu'il offre une performance et un spectacle lorsqu'il est en compagnie. Par conséquent, il se redresse, il parle et il apprend à aimer et à mentir

6. Pour l'étude de l'ingénu selon Marivaux, voir Charles Mazouer, Le personnage du naïf dans le théâtre comique, du Moyen Âge à Marivaux, Paris, Klincksieck, 1979.

7. En ce qui concerne Mme de Murat, le thème de l'emprisonnement se rapproche de l'exil et de l'assignation à demeure à Loches qui lui sont imposés pendant de longues années sur un chef d'accusation de lesbianisme. Ses appels resteront sans réponse de la part de son mari. Par ailleurs, sur l'engagement du corps et de la liberté pour Marivaux, voir Mathieu Brunet, "Quand le corps dit la liberté. Réflexions sur Marivaux et Rousseau", dans Clotilde Thouret et Lise Wajeman (éd.), Corps et interprétation (XVI $-\mathrm{XVIII}^{e}$ siècles), Amsterdam, Rodopi, 2012, p. 107-117. 
selon une séquence pratiquement instantanée. Le mensonge protecteur lui semble une ressource évidente, car il comprend d'un coup sa situation, la contrainte que lui impose la fée, et le pouvoir qu'il détient sur celle qui attend de lui plus que son obéissance et sa coopération. Le secret affecte donc la surveillance et le surveillé.

\section{Le secret comme contrainte}

La surveillance de la fée est discrète (invisible), car elle se fait par effraction à la décence et à ses propres engagements. D'ailleurs, la fée de Mme de Murat est fratricide. Quant à la fée de Marivaux, elle a enlevé un enfant qui pleure en réclamant père et mère. À cause du rapt, elle doit assumer une fonction parentale: son désir devient donc pratiquement incestueux. Chez Mme de Murat, la fée veut que son neveu se marie à sa fille (qui lui ressemble en tous points); chez Marivaux, elle veut épouser le «beau brun » elle-même. Elle se cache de la société car elle est à la veille d'épouser Merlin, dont les pouvoirs occultes seraient inefficaces au cas où elle s'unirait fort officiellement à Arlequin: «[...] sur le champ j’en fais mon mari; cette qualité le mettra alors à l'abri des fureurs de Merlin; mais avant cela, je n'ose mécontenter cet enchanteur, aussi puissant que moi, et avec qui je différerai le plus longtemps que je pourrai ${ }^{8} »$. En attendant, elle s'expose à la colère de son fiancé et à l'opprobre public. En fait, les tiers constituent un pouvoir alternatif qui tempère celui des détenteurs de secret; surveillants et surveillés sont sous le regard de la société, aussi restreinte celle-ci soit-elle. Ainsi, Parcin ne rencontre-t-il sa belle cousine emprisonnée que sous le regard de sa gouvernante: elle tolère la présence du jeune homme car elle ne voit aucune autre chance de libération pour sa pupille. Ainsi, le serviteur de la fée marivaudienne outrepasse-t-il sa fonction de valet de comédie, qui dans la scène d'exposition offre ses remontrances au maître ou à la maîtresse: il introduit ses jugements en demandant la permission d'exposer sa condamnation touchant l'inconséquence de sa maîtresse libertine, dans un tête-à-tête qui a semblé finalement trop impertinent, puisque l'adjectif «petit» a été abandonné comme familier et irrespectueux9

8. Marivaux, Arlequin poli par l'amour, op. cit., p. 88.

9. «Ce mot petit, jugé sans doute trop familier, disparaît dans l'édition N.T.I. de 1730 et dans les éditions suivantes », selon Deloffre et Rubellin (ibid., note 2, p. 1036). 
En effet, Trivelin se permet de «dire ici [s]on petit sentiment»: «[...] c'est que vous enlevez le jeune homme endormi, quand peu de jours après vous allez épouser le même Merlin qui en a votre parole»; «franchement, si vous les prenez tous deux, suivant toutes les règles, le second mari doit gâter le premier ${ }^{10} »$. Le couple que la fée formerait avec Merlin est compatible. Elle lui plaît et il lui plaisait encore tout récemment. Cependant, à la vue d'Arlequin endormi sous les arbres, la fiancée a perdu tout intérêt relatif à son union à Merlin. Lattrait que la fée éprouve pour le «bel imbécile» (l'expression est de Trivelin, mais la fée y souscrit) est donc pour un corps: «[...] la figure du jeune homme en question m'enchante; j'ignorais qu'il eût si peu d'esprit ${ }^{11}$ ». Trivelin accuse sa maîtresse de bassesse dans ses désirs. La fée ne se dédit pas. Elle aime l'homme endormi, elle croit pouvoir passer outre son manque d'esprit, mais elle cherche tout de même à contrôler sa gestuelle peu spectaculaire, puisqu'elle lui a donné fort inutilement un maître à danser. Lors même qu'il acquiert de l'esprit, " poli par l'amour », Arlequin garde un franc-parler, des maladresses, et des désirs prosaïques. En avouant qu'elle le préfère à un cadeau grossissant son cheptel de moutons, Silvia est mieux assortie à la sensibilité d'Arlequin, qu'elle ne cherche donc pas à amender.

En tant qu'expression corporelle de maîtrise, la danse d'Arlequin révèle quelque chose sur sa nature primaire, qui reste incompatible avec la fée. Arlequin poli par l'amour serait le pendant incomplet et inverse de La double inconstance où un couple aimant se fait diviser par les machinations d'un homme de pouvoir, et reconstituer dans un quadrille vers de nouveaux couples qui répondent mieux à la nature de chacun selon une gradation esthétique des sensibilités. L'excès de contrainte est ce qui révèle le caractère résolu et la nature d'Arlequin. Sur un mode mineur et purement stratégique, c'est bien là l'illustration de la réflexion de Mme de Merteuil à l'endroit des désirs imprécis de Danceny pour Cécile : il lui faut des obstacles et des contraintes pour se proposer directement à la jeune demoiselle. Car l'interdiction de revoir Silvia conduit Arlequin droit à son mariage avec cette bergère. Ainsi, la pièce de Marivaux empêche la fiancée de Merlin d'abuser d'un jeune homme (de s'approprier son corps et d'en abuser comme le

10. Ibid., p. 87 et 88 .

11. Ibid., p. 89 et 88 . 
recommanderait Sade), d'autant plus que l'attachement naturel d'Arlequin est dirigé vers une jeune bergère impatiente de former un couple. C'est que la contrainte «positive» de «la double inconstance» est légitimée par la division de deux amants pour rendre quatre personnes heureuses, tandis que le désir forcené de la fée d'Arlequin poli par l'amour reste scandaleusement illégitime par manque de symétrie. Lunion qu'elle projette avec son prisonnier est d'abord plus ou moins scandaleuse par la configuration de la pièce qui la pose en adulte devant un enfant dont elle veut détourner l'éveil sensuel et identitaire. Ensuite, la bourrée n'est pas le tempo qui convient à la fée. Cette dernière est châtelaine alors qu'un Arlequin (par la nature du personnage type qu'il représente) est tout au plus un bûcheron pour s'associer à une fermière, selon Mme de Graffigny ${ }^{12}$. Pour sa part, Trivelin n'espère rien de l'esprit que l'amour pourrait prêter à Arlequin : « [...] voici un paysan qui veut vous donner le plaisir d'une danse de village, après quoi nous irons manger ${ }^{13} »$. La bourrée de la troisième scène qui provoque ce commentaire se retrouve dans le divertissement de clôture de l'action théâtrale, lorsqu'Arlequin célèbre son amour avec Silvia: « [...] je veux qu'on chante, qu'on danse, et puis après nous irons nous faire roi quelque part ${ }^{14} » . \grave{A}$ «Roy», Furetière confirme bien une expression indiquant qu'il s'agit de payer un festin ${ }^{15}$. En effet, Trivelin avait prévu ce dénouement, qui est en partie son œuvre. Suivant son jugement personnel, il décide de trahir sa maîtresse et de protéger l'amour naturel et légitime entre subalternes.

12. Dans sa lettre à Devaux du 3 mars 1747, Françoise de Graffigny caractérise Arlequin comme souvent «maussade», c'est-à-dire grossier et désagréable : «Lis donc Arlequin poli par l'amour, La double inconstance et mille autres», lui enjoint-elle. Elle semble se défendre d'avoir attribué un rôle brusque à Arlequin bûcheron dans une pièce de sa composition, dans Correspondance de Madame de Graffigny, éd. English Showalter, dir. J. A. Dainard, Oxford, Voltaire Foundation, 2003, vol. VIII, lettre 1123, p. 266.

13. Marivaux, Arlequin poli par l'amour, op. cit., p. 91.

14. Marivaux, Arlequin poli par l'amour, op. cit., p. 109.

15. Antoine Furetière, «Roy», Dictionnaire universel, contenant généralement tous les mots français tant vieux que modernes et les termes des sciences et des arts, La Haye, Pierre Husson, Thomas Johnson et al., 1727, vol. IV. Il peut également s'agir de références à d'autres pièces où Arlequin est roi, comme dans la farce de Lesage, Arlequin roi de Serendib (1713), où le pouvoir fragilise et où les apparences sont trompeuses. 
En ce qui concerne la nouvelle force d'initiative exercée par Arlequin, Deloffre et Rubellin estiment que « [...] seule l'épreuve d'un sentiment dominateur révèle à l'homme le sens de son existence et la mesure de ses possibilités ${ }^{16} »$. Or le caprice et la tyrannie de la fée sur Arlequin, envers lequel elle a donc un désir immodéré et injustifiable, ne peuvent que lui attirer la honte publique, surtout si le jeune homme ne répond pas à ses souhaits. Sa tyrannie est faiblesse - elle a cédé sans réflexion ni frein à la tentation de posséder (unilatéralement) un bel endormi. Finalement, dans son rôle de surveillance secrète, c'est elle qui formule un souhait sans efficacité, et le surveillé qui est l'exécutif dont elle est à la merci. Selon l'analyse marivaudienne, le tyran qui espionne en secret est impuissant à diriger une action qu'il ne fait que suivre, et il perd son autonomie et sa liberté d'agir puisqu'il se retrouve enchaîné au sujet surveillé. Le tyran qui espionne s'en trouve symboliquement castré et second vis-à-vis de l'action. La fée avait compté sur la nature et sur l'isolement d'Arlequin dont elle canaliserait les pulsions. Mais du côté d'Arlequin, les automatismes corporels de l'amour sont moins dociles que prévu. Les libertins et la nouvelle culture du sentiment s'affrontent dans le cadre désigné de la Nature, dont les deux modes amoureux se réclament (libertin et sentimental). La position prédatrice et libertine de la fée réduit Arlequin à la contrer sans résistance apparente, lui renvoyant un mensonge de force égale à ses inquisitions. La parade prosaïque de ce jeune «naïf» est de faire semblant d'abonder dans le sens des désirs de la fée, de répondre à ses attentes, l'ayant suffisamment devinée pour lui renvoyer approximativement ce qu'elle veut entendre. Elle comprendra ce qu'elle voudra, et elle est libre d'interpréter l'effort de ne pas déplaire en celui de plaire. Arlequin se positionne dans un rapport de miroir équivoque par rapport à sa surveillante déroutée, incapable d'évaluer si le jeune homme est sous son influence et accède à ses désirs, ou use de mensonge pour y parer. Elle devient impuissante à démêler le vrai du faux dès qu'il se met à jouer de son ingénuité pour se protéger. En effet, dans la dimension de la parole mensongère, l'effort rudimentaire d'Arlequin est déjà de la dernière habileté, car l'amour lui a appris immédiatement à percevoir ses propres paroles et gestes en tant que spectateur. En un mot, Arlequin apprend à jouer masqué lorsqu'il prend conscience du

16. «Notice», dans Marivaux, Arlequin poli par l'amour, op. cit., p. 83. 
fait que le désir privé s'expose par l'échange de paroles et de pantomimes qui le livrent à son interlocutrice.

D’une part, il s'agit de prendre conscience de soi comme un être dont la liberté dépend du respect du contrat social, des mœurs, de l'aval de la société et de ses lois. L'être n'est pas auto-centré, et indépendant de l'ordre social, à l'inverse de ce qu'en pense le tyran ou l'être immature. Le «poli» d'Arlequin amoureux est de découvrir que son désir envers Silvia est soumis au bon vouloir de son amante. La fin abrupte de leur premier entretien montre que les mouvements de cette jeune personne sont réglés par un amoncellement de surveillance légitime, basé sur les devoirs ou les attentes du groupe social : il est remarquable que la bergère doive interrompre sa première rencontre amoureuse avec Arlequin - rencontre qui l'enchante au point d'en oublier son troupeau - justement parce que, ses moutons ayant échappé à son attention, elle sent qu'il est impératif d'aller les retrouver, étant donné la déception communautaire qui suivrait sa négligence par rapport au service qui est attendu d'elle, et qui prime sur son désir individuel.

D’autre part, lorsque le contrat social est traversé par l'effraction de l'intimité (en ce qui n'est pas de son ressort), l'être surveillé pratique la désinformation, et s'impose un jeu théâtral difficile à distinguer du comportement authentique: dès que Parcin apprend le projet de mariage de la belle et bonne Irolite à un comte déshonorable, il dissimule ses sentiments et plaisante en public ${ }^{17}$. La fée abusive doit découvrir la vérité: Danamo en consultant «ses livres ${ }^{18}$ » ou bien en déchiffrant les signes de la protection magique dont Parcin bénéficie (entre autres, en se renseignant sur les lieux où il disparaît grâce à un anneau); la fée d'Arlequin l'espionne dans son rendezvous galant grâce à un anneau de Gygès qui lui permet de voir sans être vue elle-même. La dissimulation de l'être surveillé est d'ordre politique puisqu'elle se constitue en résistance à un pouvoir jugé unanimement inique et antithétique à l'ordre social (hors du naturel ou extraordinaire) - ce que la surveillance «secrète » reconnaît si elle n'agit pas entièrement à découvert. De toutes façons, pour collecter l'information, la surveillance doit être secrète. D'un autre côté, pour

17. Mme de Murat, «Le parfait amour», op. cit., p. 225.

18. Ibid., p. 227. 
être coercitive, la surveillance doit s'exposer et enrôler des adjuvants plus ou moins approbateurs ou zélés. Dans le conte de Mme de Murat, les gardes de Parcin respectent leur prisonnier (ce qui implique qu'ils agissent à contre-cœur en le gardant): «[...] surtout son courage, les touchoit d'une admiration qui leur faisoit regarder ce prince comme un homme fort au-dessus des autres ${ }^{19} »$. Visiblement, le jugement des soldats n'est pas à l'avantage de leur maîtresse.

\section{La résistance: acquiescer ou disparaître sans confrontation}

Un passage sur «les mouches» dans le Tableau de Paris, par Louis Sébastien Mercier, intrigue ${ }^{20}$. Christophe Cave décrit l'activité fébrile de celui qui, poursuivi par des «mouches» (doublées de surveillants qui confirment que les espions s'acquittent avec zèle de leur tâche), décide de donner son adresse à ceux qui le filent: ces derniers le remercient en lui laissant savoir qu'il reste dans la résistance puisqu'il déménage «après-demainn ${ }^{21}$ ». Ou bien, dans le cas improbable où il réussirait à échapper à sa filature par «l'anneau de Gygès ", sa fuite attirerait néanmoins le constat et le rapport des lieux de sa disparition spectaculaire: «il a disparu là22». La résistance ultime serait une position de fuite annulant le rattrapage, l'acquiescement permettant tout au plus de gagner du temps.

Le secret d'Arlequin ne prend de consistance qu'à partir du moment où il contrevient aux désirs de la fée régnante par une résistance soulignée, et par son attachement au mouchoir de Silvia dont il se caresse le visage. La fée le suit grâce à son anneau d'invisibilité tandis que l'émancipation d'Arlequin (son «poli») consiste à jouer à l'imbécile (ce que la fée ne lui permet plus, le sachant maintenant doté d'esprit), puis à abonder dans le sens souhaité par la geôlière lorsqu'elle le confronte avec son bilan de surveillance: «Tenez, dans le fond, je vois

19. Ibid., p. 241.

20. C'est Christophe Cave (Université de Grenoble III) qui, à l'occasion de sa communication plénière «Critique de la surveillance et éloge du secret dans la presse clandestine et la littérature d'observation (1770-1789): autour des Mémoires secrets » (CSECS/SCEDHS, Kingston, 29 octobre 2016), nous a alerté sur cette dimension du secret dont nous retrouvons le mécanisme dans Arlequin poli par l'amour.

21. Louis-Sébastien Mercier, Tableau de Paris. Faisant suite aux éditions précédentes, Amsterdam, s. n., 1788, vol. IX. p. 308, italiques dans le texte.

22. Ibid., p. 307 . 
bien que j'ai tort; vous êtes belle et brave cent fois plus que l'autre [...]. C'est que j'ai laissé prendre mon cœur par cette petite friponne qui est plus laide que vous ${ }^{23} »$. En dépit de cette maladresse savoureuse sousentendant clairement qu'Arlequin la trouve laide, la fée saisit l'intention qu'il avait de la complimenter sans toutefois se rassurer tout à fait. En ceci, il apparaît que si le secret se révèle par la résistance, il est beaucoup plus trompeur lorsqu'il se présente sous la forme de l'acquiescement flatteur. En effet, la fée de Mme de Murat se sent déroutée et se laisse bercer d'illusion puisque la réalité que lui présente Parcin correspond à sa propre perception du monde : il devrait reconnaître la force d'attraction infaillible de sa fille (ou d'elle-même, puisque l'une est la réplique de l'autre), et il devrait sans se tromper (et donc sans la tromper) la distinguer de sa préférence comme il le prétend - le temps de faire ses préparatifs de fuite. Néanmoins, la fée de Marivaux veut s'assurer que le remords apparent d'Arlequin, qui flatte ses espoirs et conforte sa perception d'elle-même et de sa position dans le monde, soit cimenté par sa participation zélée à une mise-en-scène à laquelle il obéisse sans faillir. Ne sachant où commence la réalité vis-à-vis de son désir, elle prétend étayer la réalité en faisant jouer aux deux amants un rôle qui les sépare. Cette démarche n'est pas absolument surprenante de la part d'une fée - elle mêle l'illusion à la réalité pour «réaliser» ses souhaits. Lamendement de la réalité demande néanmoins la conscience de la fausseté relative du pouvoir du monde magique, du pouvoir en général, en ce qu'il rencontre moins la vérité qu'il ne modèle son ombre.

De fait, la mise-en-scène de son propre corps démontre que la fée cherche une esthétique de la relation amoureuse, médiatisée par le vêtement, la musique, le spectacle partagé, et suscitant une émotion commune et un élan réciproque. En effet, endoctriné par les spectacles qu'elle lui offre, Arlequin devrait reconnaître l'héroïne d'opéra en la fée et son amant désigné en lui-même. Loin d'être converti à l'aide des arts, Arlequin s'endort, ou menace de « dévorer » la main de sa geôlière sans plus de formes. La fée est en même temps libertine et sentimentale - dans la prédation unilatérale et rêvant de réciprocité formelle ou substantielle. Un certain procès se profile contre le libertinage qui ne donnerait de liberté qu'à la jouissance du corps, des corps 
dépourvus d'identité. L'identité se dessinerait à travers les enthousiasmes et espoirs personnels saisissables immédiatement, sans qu'il soit nécessaire de faire intervenir de spectacle stratégique qui fournisse un modèle à ces élans. La fée attend une autre performance que le simple reflet ou l'obéissance de la part d'Arlequin - son «poli », et si l'on en croit le titre, son amour. L'espionnage de la fée commence lorsque son hôte découvre en effet ce qu'il ressent, ce qu'il est, son humanité et sa parole, dans une nature diurne, où les amants sont désireux de former un des couples du village. Ce n'est ni dans le retrait du château, ni dans celui de la salle de musique ou de spectacle féérique (qui sont du domaine de la fiancée de Merlin) que l'amour se découvre pour Arlequin. C'est dans les champs de l'idylle pastorale. La dissimulation des intérieurs où la fée se tient cachée et l'ombre de la forêt celtique et brute qu'elle traverse ne permettent pas le type d'amour que suggère la beauté d'Arlequin.

Finalement, le secret de la fée montre aussi que son désir est contré d'emblée à cause des mœurs et des lois: il est inacceptable de se saisir arbitrairement de la liberté émotionnelle et sexuelle d'autrui - et absolument impensable de songer à une quelconque réciprocité contre le gré de prisonniers, contre leur nature et leurs préférences. D’ailleurs, Silvia ne conçoit pas de contrainte à ce sujet, et elle a la certitude que les bergers viendront lui prêter main forte. De surcroît, et c'est un sujet que Marivaux développe dans sa pièce suivante, Arlequin et la fée appartiennent à des esthétiques incompatibles. L’une est féérique, de l'ordre de la pénombre, et l'autre champêtre. C'est pourquoi pour en arriver à une conclusion heureuse selon un scénario parallèle de contrainte abusive et trompeuse, La double inconstance réconcilie la tromperie et la coercition avec les inclinations naturelles d'Arlequin (attaché aux plaisirs de la bouche) et de Silvia (appréciant ceux de l'intellect): ces amants désunis trouvent le bonheur hors du couple initial. Car les premiers couples se révèlent détonants pour les amants, lorsqu'il leur est si naturel de s'attacher à des êtres aux goûts plus fondamentalement assortis aux leurs.

Si une loi de moindre résistance est le signe de la congruité (du couple, du sujet et de son gouvernement), dans Arlequin poli par l'amour, la stratégie oppositionnelle la plus efficace serait de l'ordre de l'évitement qui devance la surveillance. La fée disparaît juste avant de se marier, tentant de battre de vitesse la surveillance de Merlin qui 
pour le moment est dans la surprise de ne pas la trouver à son logis. Arlequin abonde dans le sens de la fée pour se ménager un instant de liberté, mais pour lui, il faut immédiatement conclure des alliances spontanées.

Les secrets de tyrannie ou de résistance doivent s'exercer en dehors des lois et des mours, ou dans leur angle mort, dans ce qui n'est pas l'objet cible de vigilance immédiate. La dyade surveillant-surveillé se décline en paroles surprises ou obtenues par la force, sans qu'il soit possible d'en percer absolument le voile à cause de la propriété symbolique des mots. Les phrases sont sujettes aux retournements rhétoriques, aux quid pro quo, aux euphémismes polis, aux mensonges rusés. Arlequin doit maîtriser la parole. Il en démêle l'usage: dissimuler le réel ou bien le forger. Ainsi, selon une lecture plus psychologique, Arlequin-fils devenu adulte, loquace et sevré, s'affranchit de l'autorité parentale de la fée pour se joindre à la société (comme l'indique son mariage). L'accès au logos, phallique, temporairement décentré par la maternité, est recentré sur le fils devenu pubère. À cette étape, il est secondé par d'autres hommes, tel Trivelin. Ce dernier ne tolérait l'abus de pouvoir que contre Arlequin «enfant» ou incapable de parole. Étant devenu pleinement sujet, «le beau brun» n’est plus aliéné à la mère incestueuse qu'est la fée, et il peut se joindre aux autres adultes dans les lieux ouverts et publics.

En attendant, le langage d'amant repenti vis-à-vis de la fée reste indéchiffrable - sincère ou trompeur? Est-il endoctriné, persuadé, naturellement «corrigé », trompé, ou dans une stratégie d'évitement? La fée connaît cette opacité inhérente du dialogue amoureux, et elle jette habilement le doute sur la réciprocité de l'inclination de Silvia pour Arlequin, lorsque justement, le jeune homme vient de juger prudent de jouer cette émotion de façon convaincante vis-à-vis de sa geôlière. Ce serait le contrat de mariage joignant volonté, signature, corps et parole sous le regard de la société qui serait garant d'une certaine vérité en arrêtant les doutes ${ }^{24}$. Néanmoins, à l'ère d'une société

24. Marivaux est favorable à la clarté d'expression, tout en mettant en scène des personnages retirés derrière un masque, mais qui se révèlent lorsqu'ils sont poussés au bout de la logique de leur rôle d'emprunt, rendant leur vraie nature visible où elle était jusqu'alors invisible. Pour une discussion plus poussée de l'esthétique de visibilité de Marivaux, voir Servanne Woodward, Explorations de l'imaginaire de la représentation au dix-huitième siècle français: Chardin, Vigée-Lebrun, Diderot, Marivaux, Lewiston, 
sans divorce, le mariage reste une mince garantie: les réflexions de Marivaux sur le couple dont la femme est «dans un négligé des plus négligés, tranchons le mot, dans un négligé malpropre ${ }^{25}$ », et son époux «dans un déshabillé d'une malpropreté si dégoûtante, qu'il faut assurément qu'il l'ait étudiée pour y parvenir ${ }^{26}$ », indiquent que dans le mariage, il est important de maintenir dans la durée un comportement poli de séduction l'un envers l'autre, en se rendant visible l'un à l'autre de la manière la plus engageante possible. Marivaux poursuit: «Vous me dites qu'aujourd'hui ces gens-là ne s'aiment plus, c'est qu'ils ne le méritent plus ! [...] ceux-là doivent se haïr, se trouver pis que laids; oui

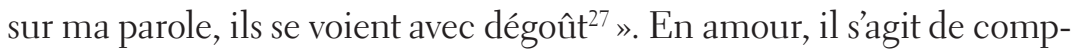
ter avec le goût, l'une des composantes de l'esthétique, et de se rendre visible plutôt que d'oblitérer sa beauté au point de ne pas être présentable en public: "Pour moi, mon cher, malgré l'embarras de cette dame, je l'ai beaucoup examinée et je vous avoue qu'elle doit être une des plus aimables femmes du monde quand elle veut l'être; car j'ai deviné ses charmes plus que je ne les ai vus. Je ne l'aimais point du tout comme elle était, mais elle me plairait beaucoup comme elle peut devenir ${ }^{28}$ ». Selon l'intention de celui ou celle qui le porte, le négligé cache l'être véritable ou l'expose dans sa splendeur et dans sa vérité. La langue agit de même, masquant, embellissant, trompant, révélant les réalités de l'être qu'elle recouvre.

\section{Logos et savoir}

Si la parole naît de l'émotion, elle est aussi le mode d'expression de la rationalité, c'est-à-dire de la mesure et du raisonnement, et de l'adhésion ou prise de parti. Mais elle est ambiguë quant au savoir. C'est

The Edwin Mellen Press, 2001, p. 203-208, et George Evans, «Marivaux’s Arlequin poli par l'amour and the Implications of Play-Acting», dans Derek Connon et George Evans (éd.), Essays on French Comic Drama from the 1640 to the 1780's, Berne, Peter Lang, 2000, p. 117-126. Sur le rapport de l'écriture engageant le corps, voir Maria Teresa Gómez Ramos, «Arlequin poli par l'amour de Marivaux: écriture et langage théâtral », Cuadernos de Investigación Filológica, 35-36, 2009-2010, p. 249-265.

25. Marivaux, Journaux et oeuvres diverses, éd. Frédéric Deloffre et Michel Gilot, Paris, Garnier, 1988, p. 200.

26. Idem. Ce négligé est indice de «guerre». Le négligé coquet est séducteur (ibid., p. 28).

27. Ibid., p. 201.

28. Ibid., p. 200. 
surtout la parole de l'engagement et du lien social qui prime, quelles que soient les réalités émotionnelles qu’elle recouvre. Ainsi Marivaux dépasse la réserve intérieure, dite de «l'honnête homme», qui reste insondable et impossible à authentifier - car l'être est devancé par une parole qui tout à la fois l'expose ou l'engage, sans qu'une forme identifiable puisse lui être assignée dans la durée ${ }^{29}$. L'être n'est pleinement visible, à ses yeux comme à ceux des autres, qu'occasionnellement, lorsqu'il sent l'urgence d'intervenir (souvent verbalement) dans le déroulement de l'action qui manifestement l'engage.

Un élément curieux de la pièce est le marché que passe Arlequin avec la fée. Depuis qu'il s'est mis à parler, il s'est rendu compte que l'échange verbal réserve de très mauvaises surprises. Alors que la fée cherche à évaluer les paroles d'Arlequin par son comportement, son attitude, en recourant au raisonnement, Arlequin trouve une autre voie pour s'assurer du sens non équivoque des mots. Pour garantir qu'il pourra découvrir les vrais sentiments de sa bergère sans être surveillé, sans qu'elle subisse de contrainte ni de punition, il opte pour le serment: «LA FÉE : - Je me retirerai. ARLEQUIN : - La peste! vous êtes une sorcière $[. .$.$] vous êtes au milieu du monde et on ne voit$ rien [...] faites un serment que vous n'y serez pas en cachette ${ }^{30} »$. La simple exposition de la surveillance change la configuration de la relation de domination, car la confrontation engage des pourparlers, des conditions, des limites, des échanges de promesse. Le «beau brun» lui a fait jurer par le Styx, à elle, la fée qui ne respectait pas sa parole visà-vis du puissant Merlin, et qui doit maintenant respecter celle qu'elle donne à Arlequin selon la formule qui lie les dieux depuis l'antiquité grecque. Déjà la fée n'ose plus exercer sa tyrannie. La relation devient contractuelle, limitée, mesurée - elle se civilise. Chez Marivaux, les pièces qui se déroulent dans des lieux isolés sont proches d'une réflexion sur le contrat social, les diverses expressions du pouvoir, et leurs circonscriptions ${ }^{31}$.

29. Le thème du secret accentue un phénomène Marivaudien observé depuis longtemps, par exemple dans Felicia Sturzer, «The Philosophical Basis for Marivaux's Hypocrite», French Literature Studies, 6, 1979, p. 56-65.

30. Marivaux, Arlequin poli par l'amour, op. cit., p. 103.

31. Henri Coulet, "Le pouvoir politique dans les comédies de Marivaux», L'information littéraire, 35, 5, 1983, p. 190-195. 
Notons au passage que l'incartade de la fée avec Arlequin est permise parce que ni Merlin, ni le reste de la société ne savent ce qu'elle entreprend. Cependant, le secret semble toujours plus ou moins connu et soutenu par un cercle restreint de familiers qui le tolèrent. Pris de pitié pour les «tendres amants », Trivelin se désolidarise de sa maîtresse et change d'allégeance, ce qui est permis par le fait que le secret d'État comme celui de la résistance au gouvernement a toujours une teneur illicite: «aussi bien ne mérite-t-elle pas qu'on la serve, puisqu'elle est infidèle au plus généreux magicien du monde, à qui je suis dévoué32 ». Celle qui brise sa parole ne mérite pas qu'on la lui tienne. Puis, Trivelin organise une véritable mise-en-scène, distribuant les rôles à jouer pour tromper la fée, lui prendre sa baguette pour la castrer de tout pouvoir, mettre l'asservissement de la fée au bon plaisir d'Arlequin en évidence, lorsque ce dernier, entièrement émancipé, pourra choisir sa destinée.

Partager le secret de surveillance, c'est choisir sa position par rapport à un pouvoir. La défection est toujours possible. La surveillance est déjà en soi un aveu d'impuissance ou de fragilité. Lorsqu'elle est révélée, Arlequin négocie une position d'allié, bien qu'il soit toujours potentiellement résistant, ce qu'il révèle assez en posant des conditions. De fait, il est dans le secret de la fée, un autre de ses adjoints. Comme eux, il a la liberté de changer de bord et de se ranger sous une autorité contraire. N'ayant pas les mêmes enjeux émotionnels, Trivelin utilise son libre arbitre contre les plans de sa maîtresse. Maintenant que l'otage a découvert l'usage et l'utilité de la parole, tout comme Trivelin, il se joint à une majorité de voix: la fée est défaite au moins par trois contre un et y perd son «sceptre», sa baguette magique.

Dans la conjuration, les détenteurs de secret détectent une fracture sociale qui concerne la représentation de soi, et sur laquelle ils évoluent comme des acteurs, avec dextérité et en double registre, tandis que les détenteurs de pouvoir jouent stratégiquement pour les contrer mais avec moins de duplicité. Ainsi, surveillants et surveillés évoluent en schisme par rapport au corps social. Que la filature soit insolemment ouverte par un pouvoir tyrannique, ou bien gardée secrète du public et des intéressés, elle a tendance à fomenter de puissants contrecourants de complots libérateurs. Ces derniers semblent se réclamer

32. Marivaux, Arlequin poli par l'amour, op. cit., p. 106. 
d'une légitimité démocratique contre les abus. L'anneau de Gygès auquel Marivaux fait allusion nous reporte à La république de Platon, qui aborde l'iniquité des forts contre le sentiment naturel de justice qui serait le partage et le recours des faibles. Marivaux semble indiquer que le pouvoir tient au consensus gouvernemental soutenu par un sentiment général de justice naturelle. 